\title{
Paediatric Brain Monitoring with Information Technology (KidsBrainIT) - ERA-NET NEURON Grant
}

\author{
C.A. Apetrei ${ }^{1}$, C. Gheorghita ${ }^{2}$, A. Tascu $^{3,4}$, A.St. Iencean ${ }^{1}$, Tsz-Yan \\ Milly Lo ${ }^{5}$, Ian Piper6, St.M. Iencean ${ }^{1,7}$ \\ ${ }^{1}$ Neurosurgery, "Prof. Dr. N. Oblu" Clinical Emergency Hospital Iasi, ROMANIA \\ ${ }^{2}$ Neurosurgery, "Sf. Maria" Children Clinical Emergency Hospital Iasi, ROMANIA \\ ${ }^{3}$ Neurosurgery, "Bagdasar-Arseni" Clinical Emergency Hospital Bucharest, ROMANIA \\ ${ }^{4}$ Neurosurgery, "Carol Davila" University of Medicine and Pharmacy Bucharest, ROMANIA \\ ${ }^{5}$ University of Edinburgh (Child Life \& Health) / Royal Hospital for Sick Children (Paediatric \\ Critical Care Medicine), UK \\ ${ }^{6}$ BrainIT Group Co-ordinator, Principal Health Care Scientist, Neuro-Intensive Care \\ Monitoring Research, UK \\ ${ }^{7}$ Neurosurgery, "GrT Popa" University of Medicine and Pharmacy Iasi, ROMANIA
}

\begin{abstract}
The complete name of this ERA-NET NEURON Grant is "Paediatric Brain Monitoring with Information Technology (KidsBrainIT). Using IT Innovations to Improve Childhood Traumatic Brain Injury Intensive Care Management, Outcome, and Patient Safety". The Project Coordinators are Ms. Dr. Tsz-Yan Milly Lo (Consultant Paediatric Intensivist and Research Lead in Paediatric Critical Care Medicine ) and Ian Piper from University of Edinburgh, UK and the partners are: Prof. Bart Depreitere and his team from Neurosurgery \& Intensive Care Research Group, University Hospitals Leuven, Belgium; Prof. Juan Sahuquillo and his team from Department of Neurosurgery, Vall d'Hebron University Hospital, Barcelona, Spain and the Romanian team with doctors CA Apetrei, C Gheorghita and A Tascu as principal investigators in three different hospitals. This material is based on the scientific project proposal with the basic project data. The aim of this grant is to test two clinically relevant hypotheses: after sustaining traumatic brain injury (TBI), paediatric patients with a longer period of measured cerebral perfusion pressure (CPP) maintained within the calculated optimal CPP (CPPopt) have an improved global clinical outcome and better tolerance against raised intracranial pressure (ICP). Paediatric TBI patients requiring intensive care are recruited from more contributing centres in 4 different countries. Their anonymised routinely collected bedside physiological monitoring data in minute-resolutions linking with anonmyised clinical and outcome data are exported
\end{abstract}


and archived in the central KidsBrainIT data-bank. CPPopt is calculated and ICP dose-response analyses are performed on the KidsBrainIT dataset and their correlations with global outcome at 6 months are determined. The final aim of this study is to improve the treatments of the abnormal physiology insults: increase pressure from brain swelling (raised ICP) and brain perfusion pressure (CPP).

Key words: cerebral perfusion pressure, children brain trauma, intracranial pressure, paediatric brain monitoring

\section{Introduction}

As we have already mentioned this material is based on the scientific project proposal with the basic project data, made by Dr. Tsz-Yan Milly Lo.

Traumatic brain injuries in children represent a major cause of morbidity and mortality worldwide and it is the main cause of death in children older than one years of age. As shown in the project proposal the "majority of children who survives a life threatening brain trauma have new disabilities that affect how they function throughout the rest of their lives. This also has great impact on their carers and societies. Currently the best option to improve survival and recovery of children with life threatening brain trauma is to improve their early hospital treatments including intensive care because none of the new experiemental therapies tested in the laboratory is useful in clinical practice."

Also we find that "the current best therapeutic option to improve paediatric TBI outcome is to optimise physiological support in the intensive-care to minimise secondary physiological insults which are proven to negatively affect outcome. However therapeutic thresholds for abnormal physiology vary between units and are implemented clinically without validation. To give these patients the best possible recovery, we urgently need clinically relevant and readily translatable research that optimises paediatric brain trauma treatment and reduces inequality between different centres."

Secondary ischaemic injury from reduced brain perfusion is the main insult neurointensive care management aims to prevent and we need to monitor cerebral blood flow (CBF) and metabolism continuously to prevent brain ischaemia. "Intact cerebrovascular autoregulation means having the ability to maintain a stable brain perfusion for varying mean arterial blood pressure (MAP). In clinical practice, we know cerebrovascular autoregulation is intact when a patient retains the pressure active pattern while considering the relationship between MAP and ICP. Optimal CPP (CPPopt) is the CPP level that maintains the pressure active pattern. Cerebrovascular autoregulation is known to be impaired after severe TBI and the degree and range of this dysfunction vary between patients and over time within the same patient. Continuous real-time physiological monitoring is a recognised standard in TBI intensive-care 
management and ICP-lowering therapy is recommended when ICP is elevated above 20 $\mathrm{mmHg}$ or more, but this treatment threshold is only based upon clinical experience." We hypothesize that having measured CPP within calculated CPPopt provide better tolerance to raised ICP and improve recovery in childhood brain trauma.

\section{Methodology}

\section{Patient recruitment:}

Children aged 2 to 16 years who require intensive care management after sustaining accidental TBI are eligible for inclusion.

The contributing units ( UK, Belgium, Spanish, and Romanian centres) have similar treatment protocols, which include:

- defined raised ICP treatment guidelines using osmo-diuretics as a first line medical treatment;

- using intravenous infusions of vasopressive drugs (noradrenaline infusion being the first choice) to drive mean arterial blood pressure to achieve a target CPP;

- sedation and muscle relaxant protocol;

- mechanical ventilation to control $\mathrm{PaCO} 2$ to low normal values;

- actively controlling core body temperature to normothermia.

\section{Data collection:}

Patients' anonymised clinical data are collected; it includes the cause and nature of injury, age, Glasgow Coma Score (GCS) on admission and after acute non-surgical resuscitation, pupillary responses, initial radiological and computerized tomography
(CT), operative and other treatment details. Each patient's anonymised clinical data is linked to their physiological and outcome data in the KidsBrainIT data-bank using an anonymous study ID. Routinely measured physiological data in minute-resolutions are captured from the bedside monitors prospectively. All physiological data are anonymised prior to exporting, and then stored in the KidsBrainIT central data-bank

Outcome assessments are performed by each local team.

\section{Data Analyses}

CPPopt calculation and ICP doseresponse visualisation analyses are performed to test our hypotheses and determine if TBI patients with favourable outcome have longer periods of measured CPP within the calculated CPPopt ranges and an enhanced tolerance of raised ICP.

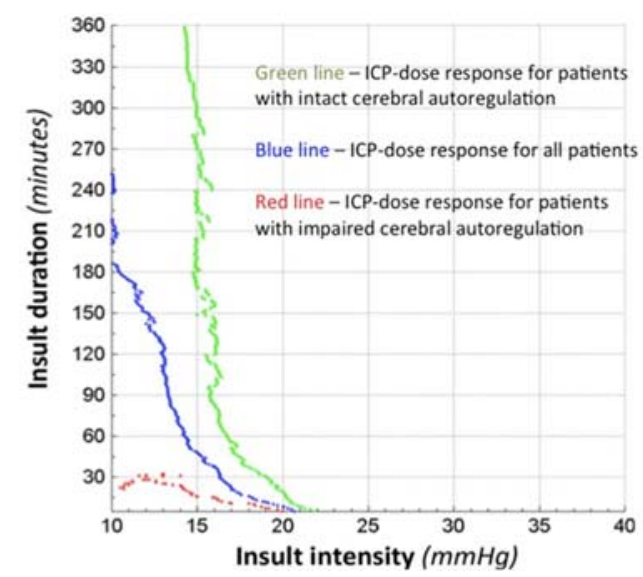

Figure 1 - Correlation of cerebro-vascular autoregulation and raised ICP 
The accordance between CPPopt and actual CPP is calculated to evaluate the association with survival $(\mathrm{GOS}>1)$ and favorable outcome $(\mathrm{GOS}>3)$ :

- the percentage of time for which the actual CPP is within the recommended CPPopt range;

- the average difference between actual $\mathrm{CPP}$ and CPPopt;

- the average absolute difference between actual CPP and CPPopt;

- the average absolute difference when actual CPP is outside CPPopt range;

- the average difference where actual CPP is below CPPopt;

- the average difference where actual CPP is above CPPopt; and

- the previous criteria are used in a multivariate logistic regression model.

\section{Conclusions}

The findings from our study and any treatment target recommendations are directly transferable back to a wider clinical audience because no special equipment or software is required beyond that is currently used for the routine minute-by-minute physiological bedside monitoring.

\section{Acknowledgments}

This work is within the grant: "Paediatric Brain Monitoring with Information Technology

(KIdsBrainIT): Using IT Innovations to Improve Childhood Traumatic Brain Injury Intensive Care Management, Outcome, and Patient Safety", grant: COFUND-NEURON III ERANET - KidBrainIT, funding no.2 / 01/06/2017.
Correspondence:

A. Tascu, "Carol Davila" University of Medicine and Pharmacy Bucharest, Romania

E-mail: tascu_alexandru@yahoo.com

\section{References}

1. Tsz-Yan Milly Lo. Paediatric Brain Monitoring with Information Technology (KIdsBrainIT): Using IT Innovations to Improve Childhood Traumatic Brain Injury Intensive Care Management, Outcome, and Patient Safety. Proposal Application Form - ERA-NET NEURON, 2016

2. Guidelines for the acute medical management of severe traumatic brain injury in infants, children, and adolescents (Second Edition). Pediatr Crit Care Med 2012. 13, No 1 (Suppl.)

3. Chambers IR, Jones PA, Lo TYM et al. Critical thresholds of intracranial pressure and cerebral perfusion pressure related to age in paediatric head injury. J Neurol Neurosurg Psychiatry 2006. 77(2): 234240.

4. Depreitere B, Güiza F, Van den Berghe G, Schuhmann M, Maier G, Piper I, Meyfroidt G. Pressure autoregulation monitoring and cerebral perfusion pressure target recommendation in severe traumatic brain injury patients based on minute-by-minute monitoring data. J. Neurosurgery 2014 Jun; 120(6): 1451-1457.

5. Güiza F, Meyfroidt G, Lo TYM, Jones PA, Greet Van den B, Depreitere B. Continuous optimal CPP based on minute-by-minute monitoring data: a study on a pediatric population. Acta Neurochir 2015.

6. Guiza F, Depreitere B, Piper I et al. Visualizing the pressure and time burden of intracranial hypertension in adult and paediatric traumatic brain injury. Intensive Care Medicine 2015. 41(6): 1067-1076.

7. Hutchison JS, Frndova H, Lo TYM et al. Impact of hypotension and low cerebral perfusion pressure on outcomes in children treated with hypothermia therapy following severe traumatic brain injury: a post hoc analysis of the Hypothermia Pediatric Head Injury Trial. Dev Neurosci. 2010; 32(5-6): 406-12 